\title{
LA CAPTURE DE L'ÉMOTION : PORTRAIT ARTISTIQUE ET PHOTO DU PAPARAZZI
}

\begin{abstract}
Anne Beyaert-Geslin ${ }^{1}$
L'article examine la notion de présence de la sémiotique en la référant à la photographie. Contrairement à la peinture, la présence photographique est nécessairement une co-présence. Le photographe est susceptible, non seulement de recueillir une émotion du modèle mais aussi de la susciter. Cette interaction est rapportée à des portraits artistiques de Laura Henno puis aux photographies de paparazzi. Elle permet alors d'esquisser une typologie fondée sur le critère de la distance.
\end{abstract}

Les études du portrait n'ont cessé d'invoquer la notion de présence en se référant invariablement à Alberti (1435/1992, p. 131) qui luimême cite déjà la formule célèbre de Pline l'Ancien : " La peinture a en elle une force tout à fait divine qui lui permet non seulement de rendre présents, comme on dit de l'amitié, ceux qui sont absents, mais aussi de montrer après plusieurs siècles les morts aux vivants $»^{2}$. $\mathrm{Si}$, confirmé au long des siècles, cet effet de présence obtient le plus large consensus, il n'a guère été rapporté au support du portrait pour

1 Anne Beyaert-Geslin est Professeur en SIC (sémiotique), à l'Université BordeauxMontaigne et Directrice-Adjointe EA 4426 MICA et responsable axe COS.

2 Pour cette référence, Spencer, J.R. (1957), Ut Rhetorica Pictura : A Study in Quattrocento Theory of Painting, Journal of the Warburg and Courtauld Institutes, 20, pp. 34-35, renvoie, non à Pline l'Ancien, mais à Cicéron, De amicitia, VII, 23.

Recherches en communication, $\mathrm{n}^{\circ} 41$ (2014). 
distinguer la photographie de la peinture, par exemple. La précision est pourtant essentielle car elle met en évidence deux particularités de la présence photographique. En premier lieu, elle confirme que, pour la photographie plus encore que pour la peinture, la présence doit être considérée comme une force à rapporter au mouvement existentiel (Merleau-Ponty, 1994, p. 160). Dans le prolongement, elle témoigne ensuite de la nécessité d'une co-présence : non seulement le sujet du portrait photographique est passé devant l'objectif, il « a été » (Barthes, 1980) mais, prolongeant cette « thèse d'existence » (Schaeffer, 1987), un photographe a nécessairement vu ce sujet ${ }^{1}$. Pourtant le simple principe d'une coprésence reste insuffisant, à moins de considérer une présence susceptible de réagir à une autre et, sous cette pression, de modifier son apparence. La présence devient ainsi une épreuve de forces. Dans la continuité, la photographie aurait un accès privilégié aux affects en raison d'un lien particulier au corps propre. Elle établirait une communication entre les deux corps propres en présence, une relation charnelle et, par une sorte de prémonition, pourrait sentir et susciter un affect tout en lui offrant un plan d'inscription pour en conserver la trace (Galinon-Mélenec, 2011). Le medium photographique a la capacité de saisir et d'enregistrer ce que ressent un corps lorsqu'il affronte celui du photographe. Il redouble en somme l'impression : celle, émotive, qu'un corps produit sur le corps photographié et l'impression figurative, autrement dit la trace ou l'empreinte que cette réaction laisse dans l'image. Si, évoquant une " prémonition du corps de l'autre », ce rapport de forces rappelle certaines leçons de la phénoménologie ${ }^{2}$, il laisse cependant un certain nombre de questions en suspens. La première question souligne que certaines photographies semblent échapper à cette règle générale de la double impression, comme l'autoportrait ou le photomaton qui en est une version particulière. Si ce regard sur soi joue de l'immédiateté de la transformation qu'autorise précisément la co-présence photographique, ce cas particulier risquerait de nous distraire de notre discussion. Ensuite, ce rapport de forces montre que, si le portrait est une « fabrique de soi » (Wrona, 2012), le portrait

1 Ce n'est pas nécessairement le cas du portrait peint. En suivant les remarques de Sorlin, on ferait valoir que le passage du modèle dans l'atelier du peintre n'est, non seulement pas indispensable à la réalisation du portrait mais ressortit en certains cas à un rituel, des peintres aussi illustres que Matisse ayant dessiné leur modèle à peu près de mémoire (Sorlin, 2000).

2 « La leçon de la phénoménologie, c'est que le corps propre est prémonition d'autrui »: mon corps n'est pas uniquement le mien mais « relation charnelle avec l'autre » indique Coquet (2007, p. 95). 
photographique construit une identité sous emprise, déjà déterminée par un vis-à-vis. Mais l'instance du portrait réagit-elle à n'importe quel corps ou à celui qui est doté d'une identité actorielle particulière, en l'occurrence d'un photographe ? Et cette instance du portrait est-elle elle-même un simple quidam ? L'enjeu de cette double actorialisation est un face à face identitaire et statutaire qui déplace l'attention d'une sémiotique du texte, attentive au plan d'expression de la photographie, vers la pratique photographique et le modus operandi de l'interaction. Déterminée par le statut des deux instances co-présentes, la pratique s'apparente alors à une traque visant la capture. Une autre question porte sur le statut de cet affect que le photographe s'efforce de manifester. Peut-il être envisagé comme une passion (Greimas \& Fontanille, 1991) ou un éprouvé (Henault, 1994)?

Cet article s'efforcera donc de saisir le "supplément sensible » qui est produit et pas seulement « enregistré » par la photographie. Il l'observera tout d'abord dans des portraits artistiques d'adolescents avant de se tourner vers un genre photographique qui est pour ainsi dire spécialisé dans la production et l'enregistrement de l'émotion, la photo faite par le paparazzi. Nous verrons ainsi comment, transformant la photographie en une épreuve de forces, la pratique modélise son plan d'expression en dramatisant la distance d'interaction.

\section{La mise à l'épreuve sensible du portrait}

Notre définition de la photographie comme «épreuve de forces » évoque d'abord les photographies au statut artistique et particulièrement les séries de portraits réalisées par Laura Henno (Summer crossing, 2008) ${ }^{1}$ et Rineke Dijkstra (Coney Island, 1992). En toute première approximation, ces photographies permettent de préciser la conception banale de la présence qui est héritière de la tradition picturale. Elles rappellent plus exactement que, dans l'image fixe, la présence se laisse décrire comme un arrêt du mouvement de l'existence. Dans ce cas, l'image immobilise en outre une transformation corporelle qui apparaît avec acuité chez les adolescentes photographiées par R. Dijkstra ${ }^{2}$ dont les formes encore enfantines contrastent avec des jambes démesurées. La présence saisit en outre un espace-temps qui assure la stabilisation

1 La série a fait l'objet de plusieurs expositions, la plus récente à la Galerie des Filles du calvaire à Paris en 2013.

2 Nous pensons particulièrement à Kolobrzeg, Pologne, une photographie évoquant la Vénus de Botticelli. 
identitaire du sujet, rappelant ainsi l'importance de la représentation du temps pour la définition de l'individu (Todorov, 2004, p. 78) ${ }^{1}$. Insérés dans le temps, ces corps ne sont plus de simples signes mais des acteurs qui jouent leur vie. Les portraits interrompent donc un triple mouvement : celui de l'existence, celui du cours de la vie de ces adolescents et, posant le principe d'une énonciation qui assure le " passage des figures du monde aux figures de sens » (Ricœur dans Henault, 1994, p. 206), celui de l'espace-temps de l'image. Il serait sans doute utile de reprendre une analyse faite par ailleurs (BeyaertGeslin \& Lloveria, 2014) pour rapporter les deux modes de construction identitaires à des paysages distincts. On opposerait alors l'instabilité à la fois spatiale et temporelle des portraits de L. Henno, saisis en terrain indécis et « entre chien et loup », et l'arrière-plan parfaitement stabilisé des scènes de plage de R. Dijkstra photographiées en plein jour.

Mais il importe surtout de rapporter cette énonciation à une intercorporalité. L'émotion et les gestes de protection des adolescents photographiés par L. Henno rappellent en effet que la présence se définit avant tout comme " une présence à et pour des instances des corps - qui y sont sensibles, en sont affectés » (Fontanille, 1998, p. 280). Si la présence suppose une interaction, la pose photographique apparaît dès lors comme une stabilisation et une iconisation d'un certain régime d'interaction, un réglage stratégique qui articule la co-présence. L'interaction manifeste ainsi la force contenue dans ces portraits. Elle la théâtralise et, au travers de petits décalages, met en lumière ce que Klinkenberg (2000, p. 279) dénomme, avec d'autres sémioticiens, la dimension épisémiotique. Il décrit ainsi un écart entre ce que l'instance souhaitait manifester et ce qui l'est effectivement. « Signe connotatif » (Klinkenberg, 2000, p. 279), la dimension épisémiotique témoigne d'une réflexivité de l'instance d'énonciation qui, capable de jugement, peut mesurer l'écart entre l'attendu et le manifesté. En ce sens, elle témoigne d'une expertise qui, au travers de la figure transgressive, met au jour une norme implicite et, fixant ces deux repères, permet de mesurer l'amplitude du mouvement de la pratique.

S'agissant du portrait, le concept d'épisémiotique traduit donc une dérégulation du portrait et l'étrangeté à soi qui se manifeste au sujet lui-même. Si, en dévoilant une ouverture du sujet au monde, la dimension épisémiotique rencontre les transformations de l'identité

1 Voir aussi, pour une approche anthropologique de cette question Le Breton, D. (1990). 
relationnelle décrites par Ricœur (1990), l'écartement de l'idem et de l'ipse, c'est pourtant en proscrivant toute régularité. L'épisémiotique est une dérégulation qui conserve cette apparence de désordre que Sartre associe à la manifestation de l'émotion. Dans le magnifique essai qu'il lui consacre, Sartre envisage l'émotion comme « une réaction organique diffuse (donnée) à la place d'une réaction adaptée, un désordre » (1938/1995, p. 40). L'émotion est pour lui un échec, une « conduite d'échec » ou plutôt une « absence de conduite » face à l'inconnu. Faisant le lien avec la définition modale de la conduite (Fontanille, 2008), sa description nous permet d'apercevoir une incapacité du sujet à articuler une réponse par les modalités et avant tout, à la construire sur le vouloir qui régit la séquence de la conduite. En somme, le sujet ému serait privé de pouvoir vouloir.

Le désordre de l'émotion est en l'occurrence suscité par un modus operandi consistant à immerger les jeunes modèles dans un milieu inconnu afin de « faire basculer l'image vers quelque chose d'ambigu » (Champesme, 2007, p. 47). L'artiste guette alors leur réaction, le geste ou l'expression inattendus, la manifestation émotive qui trahit la perte de contrôle de soi. Mais ce dispositif de capture, fût-il aussi léger et sommaire, suffit à requalifier le principe de l'ajustement qui caractérise la pose photographique (Beyaert-Geslin, 2013) en manipulation. Loin de s'ajuster à son sujet en participant à la co-construction énonciative, le photographe lui impose son intentionnalité. Les deux figures symétriques de l'interaction définies par Landowski (2005) ne suffisent pourtant pas à caractériser cette traque photographique qui guette avant tout l'aléa : L. Henno ayant posé son cadre et immergé son modèle dans un environnement, elle laisse l'espace et le temps agir sur lui et susciter une émotion. Avec elle, « l'énergie nerveuse se déchargerait au hasard et selon la loi du moindre effort », explique Sartre qui place de ce fait l'émotion sous les auspices de l'accident (1995, p. 14). «Elle est subie, » dit-il, « elle surprend, elle se développe selon ses lois propres et sans que notre spontanéité consciente puisse modifier son cours d'une façon très appréciable ». Sous ses apparences de simplicité, le stratagème mis en place par L. Henno inscrit donc une possibilité d'aventure au cœur de la manipulation, en laissant ou en faisant survenir comme dit Zilberberg (2006) l'expression inattendue, l'écart, l'irrégularité qui défie la programmation (Landowski, 2005) et, au travers d'un mouvement existentiel, manifeste une individualité.

$\mathrm{Si}$, dans le portrait photographique, le sourire du modèle peut apparaître comme une manifestation de cette maîtrise, une symbolisation 
d'un « meilleur de soi » présenté à l'autre (Goffman, 1973) en quelque sorte, le photographe qui guette les signes d'abandon doit proscrire le sourire. Cet effacement apparait dès lors comme la toute première prescription d'un protocole qui installe la pratique dans la durée et appelle le modèle à la concentration. C'est ce protocole que R. Dijkstra met en place pour saisir la fatigue, la défaillance, le relâchement qui suit l'effort des jeunes gens au sortir de l'arène (Matadors, 1994) ou celui des jeunes accouchées épuisées (Tecla, Amsterdam, 1994 ; Julia, den Haag, 1994) ${ }^{1}$. Bien loin des scènes glorieuses de corridas ou des sereines Nativités de la tradition picturale, ces portraits décrivent la vie comme un dur combat dont certains stigmates, marquant encore les vêtements, le visage ou le corps, font le récit. C'est comme s'ils commandaient au corps de parler et d'assumer seul la construction identitaire.

Il serait sans doute utile d'affiner la comparaison pour montrer comment les photographies de R. Dijkstra, en associant un point de vue frontal à de grandes dimensions ${ }^{2}$, construisent un dispositif spectatoriel qui pose un point de vue éloigné, mobilise toute l'attention et invite l'observateur à détailler le jeune corps présenté en maillot de bain. C'est comme si l'observateur guettait alors le commencement d'une danse. Dans les photographies de L. Henno au contraire, le regard s'approche et découvre un corps enveloppé de longs vêtements, immergé dans son espace clos et indéfini. Ici, la gestualité ouverte des adolescents-danseurs fait place à des gestes et des postures autocentrés qui dissimulent les mains sous de longues manches. Tout se passe comme si l'ouverture de l'espace invitait le corps à l'expansion et la fermeture, au contraire, au repli sur soi en déterminant deux dispositifs énonciatifs ouverts ou fermés et des modes de présences opposés.

\section{La photographie du paparazzi}

Avec les portraits d'adolescents de L. Henno et R. Dijkstra, étudiés avec Vivien Lloveria (2014) nous avons posé les conditions d'une mise à l'épreuve photographique qui, saisissant le corps alors qu'il perd le contrôle de lui-même ou s'efforce de le reprendre, manifeste un écart le donnant " lui-même comme un autre » pourrait-on dire en reformulant Ricœur (1996). Si cette quête de « l'étrangeté à soi »

1 Les deux séries font partie des collections du Musée national d'art moderne, Centre Georges Pompidou, Paris.

2 Le portrait intitulé Hilton Head Island, S.C., USA, June 24, 1992, mesure par exemple 45,7 X $60 \mathrm{~cm}$. 
nous semble emblématique du portrait artistique contemporain qui tente ainsi de saisir la fragilité existentielle, elle est le thème même de la photo du paparazzi. Dans ce cas, l'écart se systématise et peut être modélisé, comme nous essayerons de le montrer en mettant en évidence les régularités du modus operandi et leurs répercussions dans l'image. Passer du genre artistique à la photo du paparazzi suppose néanmoins qu'on décrive le changement de statut du sujet. Le paparazzi ne photographie pas des inconnus mais des célébrités. Une valeur est donc introduite dans la relation sémiotique qui indique en quoi l'objet, en l'occurrence le modèle, est « désirable » pour ce sujet et ce que celuici y investit. Elle définit alors les statuts respectifs des deux instances en présence et détermine le mouvement narratif qui les porte l'un vers l'autre (Henault, 2012, p. 157). Si cette « valeur ajoutée » détermine l'identité du modèle, elle fixe aussi le cadre modal de sa construction identitaire et en situe l'enjeu entre l'apparence (Arendt, 2007) et la visibilité (Heinich, 2012). Précisons les termes de cette transformation modale.

Pour commencer, on rappellerait que la présence convoque la phénoménologie en tant qu'étude des phénomènes, autrement dit de « ce qui se dénonce soi-même » selon l'expression de Sartre (1938/1995). La réalité de ces phénomènes se confond avec l'apparence, non que celle-ci dissimule « quelque chose derrière quoi il y aurait encore quelque chose qui n’apparaît pas » mais parce que cette dénonciation de soi doit être considérée comme unique existant. Arendt (2007, p. 40) a inventorié les contraintes de l'apparence. « Etre en vie signifie être mû par un besoin de se montrer », dit-elle en évoquant un devoir d'extériorité : « Tout ce qui est est destiné à être perçu ». Or, si les adolescents photographiés, pour ainsi dire prisonniers de leur apparence, sont soumis à ce devoir d'extériorité, il ne va pas de même des « people » qui sont en outre animés par un désir de visibilité, veulent être vus et reconnus comme l'a souligné Heinich $(2012,2014)$. Le cadre des modalités permet de saisir la nuance : l'apparence relève d'un devoir être et faire et la visibilité d'un vouloir être et faire. Loin d'être anodine, la différence permet de situer le sujet vis-à-vis de l'action en associant le vouloir à une virtualité qui le met seulement en mouvement ${ }^{1}$ et le devoir à un mode d'existence actuel qui le confronte aux résistances du monde tout en le rapprochant de la réalisation de l'action. Si la visibilité relève d'un

1 Pour Hannah Arendt aussi, le sujet débute sa construction par un « je veux ». Voir Arendt (1978/2007). 
désir sans contrainte, le paparazzi se charge donc de la réintroduire. Il ne s'agit plus d'être visible quand le modèle le voudra mais n'importe où et n'importe quand. En tout cas, selon le bon vouloir du paparazzi. Une clause aléthique et déontique (devoir être et faire) a donc modifié le contrat.

Cette clause supplémentaire transforme l'interaction, dramatisant l'énonciation et produisant ce qu'on pourrait appeler par référence à Barthes (1957) une " photo choc », non parce que son contenu produit un choc (appelons-le choc de réception) mais parce que l'interaction des deux instances produit une collision, un choc énonciatif. Loin des modèles plus ou moins pacifiques d'interaction présentés par Landowski (2005), la manipulation, la programmation, l'ajustement et l'alea, qui restent marqués par un principe de collusion dont les risques sont finalement très limités, la photographie du paparazzi se fonde au contraire sur un antagonisme. Mettant au jour le stéréotype et l'implicite de la collision, ce principe d'antagonisme rapporte l'énonciation photographique au schéma de l'épreuve de la sémiotique greimassienne. Il dévoile plus exactement les deux versants de l'épreuve qui qualifie à la fois l'image elle-même (épreuve photographique) et la pratique photographique où elle décrit à la fois le parcours du modèle, littéralement mis à l'épreuve, et celui du paparazzi qui doit traverser les diverses épreuves du parcours narratif jusqu'à l'épreuve glorifiante que constitue le vol de la photographie. Cette photographie consacre alors la réussite de la traque et vaut comme preuve de la capture.

Epreuve et preuve, la photographie du paparazzi expose aussi des preuves figuratives de la collision énonciative qui donnent consistance à la dimension épisémiotique et témoignent de l'écart vis-à-vis de ce qui apparaît désormais comme un stéréotype de la photographie, que nous avons dénommée photographie de collusion, celle qui témoigne d'un accord entre les deux instances en présence. Ces écarts caractéristiques sont ceux du flou, du bougé qui traduisent une désynchronisation des deux corps en présence, mais aussi la faible définition liée à l'utilisation du zoom qui creuse la profondeur de champ. Ces écarts récurrents permettent d'identifier le « vol photographique » et construisent, par récurrence, une nouvelle figurativité stéréotypée, une norme fondatrice du genre de la photographie du paparazzi. Les écarts épisémiotiques ont construit un nouveau modèle.

Mais en quoi consiste précisément le vol photographique ? Nous avons indiqué l'ajout d'une contrainte qui encadre désormais le désir de visibilité du modèle. Il convient de rapporter cette contrainte à une 
intrusion dans la sphère personnelle qui requalifie le visible en donnant accès à l'intimité des actions, mais aussi aux émotions tout en donnant une lecture polysensorielle du corps. Comme l'a souligné Hall (1971), la proximité associe les informations cognitives et affectives et pourrait même, si l'on en croit le peintre Maurice Grosser, donner accès à l'âme du modèle ${ }^{1}$. Cet apport permet de rapporter le vol photographique à la distance qui renégocie la profondeur de champ et enregistre les traces de l'effraction. Mais une question surgit car, après tout, ces informations cognitives et affectives pourraient tout aussi bien être obtenues sans collision, en reproduisant la distalité canonique du portrait qui correspond essentiellement à la dimension personnelle de Hall (1971). Mais un tel portrait autoriserait le principe de la pose, suivant en cela l'ajustement des deux instances que nous avons décrit par ailleurs en observant la pratique du « shooting » du mannequin qui construit une identité dans l'interaction (Beyaert-Geslin, 2013). Tout au contraire, la photographie du paparazzi doit manifester l'antagonisme, se signaler comme « volée » et afficher les signes du décalage épisémiotique. Loin des patientes séances de pose de la photo de mode qui co-construisent l'identité en mettent en évidence la polarité de l'interaction, celle du paparazzi dissimule une des instances en présence : deux instances entrent certes en relation mais, le modèle étant photographié à son insu, c'est la notion même de présence et la plasticité de l'apparence qui sont questionnées. Si la photographie de mode perfectionne l'art de la pose, de la co-construction et du façonnement de l'apparence, celle du paparazzi proscrit la pose et s'efforce de capturer l'image d'un modèle parfaitement ingénu ${ }^{2}$.

\section{Les limites distales de la visibilité}

Cette exigence n'est pas sans incidence sur la profondeur de champ de la photographie. On distingue communément la photographie volée éloignée et celle qui est prise très près, deux distances qui correspondent

1 « A la distance personnelle, l'âme du modèle commence à transparaître » (Hall, 1971).

2 Le documentaire de Raymond Depardon, Reporters (1981) présenté dans le cadre de l'exposition de Metz révèle toute l'ambigüité de la pratique du paparazzi. Le comédien Richard Gere, traqué, refuse de se laisser photographier. Les paparazzis immobilisent sa voiture. S'ensuit une vive discussion au terme de laquelle Gere consent à se laisser photographier. Ses « chasseurs » refusent alors en disant que le cadre ne convient pas. Tout se passe comme si la pratique du paparazzi excluait la possibilité d'une pose. 
à la pratique du téléobjectif ou du flash ${ }^{1}$. Ces modèles lointain et proche, qui exemplifient l'absence ou l'insistance du face-à-face, réfèrent à des pratiques française et américaine. Ils investissent les limites du champ visuel comme pour le dépasser et indiquer les deux seuils extrêmes de la visibilité. Sur ces seuils, la stratégie consiste à montrer plutôt qu'à présenter, en dévoilant une instabilité, une précarité du voir confronté à une photographie mal définie. Nous avons observé cette instabilité à propos de la photographie de reportage (Beyaert-Geslin, 2009). Nous nous référions alors à l'opposition traditionnelle en pragmatique entre les énoncés dits performatifs et les énoncés descriptifs. Manifestant une grande force prédicative, la photographie prise au plus près est un énoncé performatif mais elle devient descriptive lorsqu'elle s'éloigne. Tout se passe donc comme si la force de l'acte de langage photographique interdisait la description de son contenu et inversement, comme si la description de l'acte en affaiblissait la force illocutoire.

Cette tension entre le performatif et le descriptif, portée à son paroxysme par la photographie du paparazzi, dessine un cadre de visibilité. Elle révèle deux bornes du visible qui s'ajoutent au cadre latéral décrit par Fontanille (1989) avec ce qu'il est convenu d'appeler le schéma de la modalisation cognitive de l'espace. Avec ce modèle, le sémioticien explicite les figures d'une interaction entre un informateur (qui fait savoir) et un observateur (qui veut voir) et associe à la figure de l'exposition, l'alternative de l'inaccessibilité qui concerne tout ce qui est hors des limites latérales, et celle de l'accessibilité qui ouvre une brèche permettant d'y accéder tout de même. Si ce modèle peut être conservé à l'esprit comme un cadre général du visible, on découvre ici une prescription spécifique de la photo du paparazzi qui est déterminée, non par la latéralité, mais par la distalité. Elle associe les deux limites profondes de l'image à une modalisation seconde du voir qui mobilise toutes les ressources de l'attention et le transforme en « apercevoir» (lointain) ou en « scruter» (proche). La photographie du paparazzi dramatise ainsi le visible en repoussant son modèle tout au fond de l'image ou en l'attirant au contraire à l'avant plan, comme pour enfreindre la démarcation entre l'espace énonciatif et l'espace d'énonciation et briser ce qui serait la paroi de l'image.

1 Chéroux (2014, p. 15) oppose explicitement les deux modus operandi $:$ le téléobjectif et le flash. L'opposition est également au centre des entretiens des paparazzis, menés par Clément Chéroux, Camille Lenglois, Véra Léon et Max Bonhomme (2014, p. 32). 
En dévoilant cette polarisation distale de la photographie volée, nous soulignons le statut particulier de l'avant-plan de la photographie où se situent à la fois le producteur et l'observateur de l'image. Cet espace « hors champ » détermine à la fois le statut de l'instance photographiée dans l'énonciation, comme l'ont montré maints auteurs depuis Schapiro (2000) et Paris (1969) mais il détermine aussi, corrélativement, le régime de croyance et le genre de l'image (Eco, 1987 ; Joly, 2009). Pour Eco, l'opposition face/profil répercute celle du documentaire et de la fiction, par exemple. Suivant cette longue généalogie, on avancerait que ce bord frontal, dramatisé par la photo du paparazzi, est le point d'appui d'un discours véridictoire qui prétend livrer une certaine vérité de l'être.

Ces photographies traduisent deux façons de briser la possibilité de la pose, le contrôle de son apparence. La photographie volée éloignée surprend un sujet inconscient de l'interaction, alors qu'il est absorbé dans une action et construit une scène centrée sur lui-même bien qu'offerte à notre point de vue. Cet absorbement observé par Fried (1990) dans la peinture du XVIIIe siècle, produit un effet de sincérité, c'est-à-dire d'accord du sujet à lui-même, et d'authenticité, en traduisant une absence d'interférence entre l'image et son original (Beyaert-Geslin, 2009). La photographie volée proche surprend un sujet qui peut être conscient de l'interaction, donc une présence, mais cherche à s'y dérober. Néanmoins, à la différence de la photo éloignée qui affiche les preuves figuratives du vol, elle pourrait en être dépourvue et laisser à son modèle le loisir de la pose. Il n'est donc pas rare que le paparazzi attende la réaction à la première photographie, une réaction de défense ou d'agressivité qui sensibilisera le plan d'expression. La première photographie, souvent faite avec la violence d'un flash, vaut alors comme une provocation, une manipulation suscitant un faire-faire. Ainsi les deux limites de la visibilité correspondent-elles à un être à soi (la photographie volée éloignée) ou un être hors de soi (la photographie volée proche) et donnent lieu à deux réglages épisémiotiques distincts. Dans la zone médiane, le modèle reprend le contrôle de soi tandis que la photographie retrouve son modèle.

Il serait sans doute utile de préciser ce rapport à la véridiction. Lorsqu'elle s'efforce de saisir un sujet ingénu, abandonné à lui-même, en brisant le protocole de la pose, la photographie du paparazzi prétend dévoiler une vérité cachée, un secret, ou plutôt l'être dissimulé sous le paraître. Sa stratégie vise donc la révélation, ce qui signifie aussi qu'elle 
associe la pose à une possibilité de simulation ou de dissimulation ${ }^{1}$ du modèle. Il s'agit de dénuder et plus exactement de faire tomber le masque de la célébrité. Un peu d'attention suffirait d'ailleurs à référer les secrets qu'elle prétend dévoiler à une axiologie banale touchant au sexe, à la maladie, à la tromperie conjugale. C'est comme si elle prétendait, en dévoilant systématiquement un «mal caché », pondérer ou renier des valeurs positives par des négations systématiques. Cette photographie recherche en somme le vice ou le défaut caché comme pour saisir un « flagrant délit » en se posant en délégué d'une sorte de moralisation sociale.

Il est sans doute indispensable d'affiner cette esquisse. Dans un souci de caractérisation, nous avons souligné la brutalité de la photographie du paparazzi en signalant seulement les contrastes du proche et du lointain et d'un modèle américain ou français. Des différences temporelles pourraient pourtant être apportées. Une histoire de cette photographie permettrait alors d'apercevoir une sorte de décaractérisation dans la période actuelle, où le choc, la collision sont atténués, où les « people » semblent de moins en moins surpris par le photographe. Le régime de croyance et le statut même du modèle sont reconsidérés. C'est comme si l'enjeu n'était plus de révéler une vérité cachée ou alors, comme si le modèle était devenu complice voire commanditaire d'un jeu de la vérité qui, parce qu'elle s'efforce de saisir une apparence et masque la pose sous le voile de l'instantané, transforme cette photographie en une pseudo photo de mode.

Mais reprenons des oppositions plus fermes. Passer de la photographie artistique à celle du paparazzi nous a permis de faire le lien entre le texte et la pratique pour nous glisser dans le modus operandi photographique. L'une comme l'autre s'efforcent de déjouer la «fabrique de soi » (Wrona, 2012) du portrait en renégociant le protocole de la pose et en interférant dans le contrôle de soi. Dans les photographies de L. Henno et R. Dijkstra, la notion de durée reste essentielle, son allongement autorisant la mise au jour d'une émotion profonde ou d'un épuisement. Tout se passe comme si la durée, au lieu de donner le loisir de se refaçonner de se fabriquer une identité comme elle le fait avec la pose, laissait le corps s'exprimer et raconter seul l'identité. Il s'agit donc, là aussi, de révéler une vérité cachée, l'être dissimulé par le paraître de la sociabilité. Au demeurant, on

1 Brandt (1995) a revisité le carré de la véridiction de Greimas pour l'appliquer aux objets, et recouvre les figures du secret et de l'illusion par la dissimulation et la simulation. 
pourrait évoquer, dans la photographie artistique comme dans celle du paparazzi, une stratégie d'extériorisation par le langage du corps. Toutes deux interrogent le corps propre en tant qu'instance du passage de l'intéroceptif à l'extéroceptif. Néanmoins la brèche ouverte par le modus operandi photographique et la présence photographique transforment les postures de protection (voir les postures autocentrées des adolescents de L. Henno) en gestes de défense ou d'agressivité adressés au photographe intrusif.

Les photographies réunies par le catalogue de l'exposition Paparazzi ! du Centre Pompidou de Metz constituent un excellent corpus pour l'étude des récurrences textuelles. Elles révèlent certaines régularités figuratives frappantes, par exemple les prises de vues à l'intérieur des voitures, dans les arrière-salles et de nuit, notamment, et les tentatives de protection, d'obstruction (Fontanille, 1989) derrière ses mains avancées devant le visage. Si, associées à des focales spécifiques, ces récurrences spatiales et gestuelles construisent une figurativité stéréotypée, elles prédéterminent aussi la conversion du plastique en figuratif. Ces photographies soumettent les figures du monde au décadrage, à des contrastes lumineux extrêmement marqués qui délavent les couleurs et diluent les formes. Les photos des paparazzis sont « lessivées » par le contact des corps et le frottement de la présence. Par ce réglage épisémiotique qui met au jour tous les signes d'une effraction, elles se déclarent comme résultat d'un vol.

Cette modalisation du plan d'expression photographique permet de préciser notre esquisse véridictoire. Dans la photographique artistique comme dans celle du paparazzi, il s'agit au demeurant de manifester une vérité cachée de l'être, fût-elle stéréotypée ou non, l'écart épisémiotique dévoilant finalement l'attente d'une sorte de normalité sociale.

Dans le premier cas, on cherche à saisir une intériorité au travers d'une manifestation corporelle. L'extériorité donne accès à cette intériorité et l'état de choses à un état d'âme, en quelque sorte. Dans le second, on cherche deux choses. Soit l'effraction dans l'intimité, autrement dit dans la vie privée, qu'on réalise en surprenant le modèle « absorbé » dans des actions intimes, soit la réaction qui trahit l'émotion. On dévoile ainsi deux rapports différents aux états d'âme, l'un suscitant la production d'une émotion qui émerge dans la durée et l'autre une humeur brusque. Croisant les critères tensifs de l'étendue (en l'occurrence, la durée) et de l'intensité, ces deux figures nous rappellent la nomenclature des états affectifs de Fontanille et Zilberberg (1998, p. 213) (l'émotion, l'inclination, la passion et le sentiment) et leur 
distribution dans un schéma qui installe progressivement ces passions dans la durée. Notre corpus nous incite cependant à introduire une dérogation dans ce schéma pour décrire l'émotion dans une temporalité qui nous autorise à l'associer au mode d'efficience du parvenir en l'opposant au survenir de la manifestation d'humeur.

\section{La manifestation des passions}

Dans quelle mesure cette dimension épisémiotique révélée dans nos deux photographies réfère-t-elle à l'édifice épistémologique des passions ? Au demeurant, il faut écarter le cadre théorique dessiné par Greimas et Fontanille (1991) qui décrit, à partir d'exemplifications littéraires, de " grandes passions » telles l'avarice ou la jalousie, notamment. Les passions prennent ici un sens plus banal pour recouvrir indifféremment les états d'âmes et les humeurs. Mais une autre différence s'impose à l'attention. En effet, si cet édifice théorique se réfère à des " passions de papier » selon l'expression d'Anne Henault (1994), nos photographies scénarisent quant à elles des émotions " véritables " qu'elles ont par ailleurs suscitées. Il faudrait encore se demander, en suivant l'interrogation de Ricoeur (Henault, 1994), s'il est ici question de la passion (de l'énonciateur) ou des passions construites par le discours, éprouvées ou construites. Avec le cadre théorique de l'éprouvé, on examine un archisémème susceptible de recouvrir la diversité des affects et renvoyant à un signifié « musculaire, glandulaire, et épidermique » (Henault, 1994, p. 5). Le concept s'avère adéquat parce qu'il prospecte en amont de la lexicalisation et s'efforce précisément de saisir la perturbation avant qu'elle frappe le discours. L'éprouvé, explique encore cet auteur, recouvre

ce monde d'humeurs et d'impressions subjectives qui peuvent animer un discours, sans nécessairement que ce discours ait été prévu pour les extérioriser et les exprimer, sans même que ces impressions s'intériorisent en affects individuables ou nommables, pour celui-là même qui les ressent (Henault, 1994, p. 7).

Sur ce point, notre étude autorise donc un pas supplémentaire et témoigne d'une possibilité de manifestation de l'éprouvé. En effet, contrairement au genre littéraire contraint d'imiter (l'éprouvé est « une sorte d'immédiateté, que la création littéraire s'efforce d'imiter », 
convient Hénault (1994, p. 5)), le medium photographique peut capturer et même produire l'affect, faire éprouver en quelque sorte et en conserver la trace. Avec cette comparaison, on dévoile donc une prérogative de la photographie qui rend l'émotion observable, peut l'enregistrer mais de surcroît la produire par les jeux conjugués de la distance et de la présence.

Avec la photo du paparazzi, nous avons mis en lumière certaines prérogatives du médium photographique sur la manifestation et plus exactement, sur le passage d'une immanence à une manifestation. $\mathrm{Au}$ travers de la notion d'épisémiotique, nous avons restitué l'écart qui correspond à la manifestation d'un éprouvé au travers d'un rapport régulier entre le figuratif et le plastique. Il resterait un dernier pas à faire pour graduer la manifestation en l'associant à différentes possibilités d'extériorisation, rétensive et émotionnelle dans nos photographies artistiques, ou protensive et gestuelle dans la photo volée proche. Tandis que la photographie artistique guette l'instant où la carapace du corps propre se fendille d'elle-même, la photo volée proche la brise pour produire une réaction et une inscription dans l'image. Elle assimile alors la pratique photographique à un acte d'agression et l'appareil à une arme, ce qui était après tout sous-entendu par des théoriciens tels Barthes (1980) et Roche (1982) qui faisaient déjà le lien entre la textualité et le modus operandi, la traque photographique.

\section{Références}

Alberti, L. B. (1435, éd. 1992). De pictura, (trad. J. L. Schefer). Paris : Macula.

Arendt, H. (2007). La vie de l'esprit, la pensée, le vouloir : L'apparence II. Paris : PUF Quadrige.

Baqué, D. (2007). Qu'est-ce que la photographie aujourd'hui ? Boulogne : Beaux Arts Editions.

Barthes, R. (1980, éd. 2004). La chambre claire : Note sur la photographie, coll. Cahiers du cinéma. Paris : Gallimard, Seuil.

Barthes, R. (1957, éd. 2014). Mythologie, coll. Points essais. Paris : Seuil.

Beyaert-Geslin, A. (2009). L'image préoccupée. Paris : Hermès-Lavoisier.

Beyaert-Geslin, A. (2013). Quand le candidat devient président. Arguments pour une sémiotique de l'énonciation visuelle. Revue française des sciences de l'information et de la communication, 3. Disponible à http://rfsic.revues.org

Beyaert-Geslin, A., \& Lloveria, V. (2014). Une approche épisémiotique de la présence. Sur quelques portraits d'adolescents. Versus, 117.

Brandt, P. A. (1995). Quelque chose : nouvelles remarques sur la véridiction. Nouveaux actes sémiotiques, 39-40. 
Brochard, Y. (2011-12). Dans Summer crossing, catalogue de l'exposition Laura Henno. Paris : Filigranes éditions.

Champesme, M.-T. (2007). Laura Henno. Dans F. Barré, F. Hébel \& B. Curiger (Dir.), Les rencontres d'Arles Photographie. Arles : Actes Sud.

Chéroux, C. (2014). Treize thèses et demie sur le concept de photographie de paparazzi. Dans Paparazzi! Photographes, stars et artistes, catalogue de l'exposition, présentée au Centre Pompidou-Metz du 26 février au 9 juin 2014, pp. 11-19. Paris : Flammarion ; Centre Pompidou-Metz.

Chéroux, C., Lenglois, C., Léon, V., \& Bonhomme, M. (2014). Les risques du métier. Entretiens avec des paparazzis. Dans Paparazzi! Photographes, stars et artistes, catalogue de l'exposition, présentée au Centre Pompidou-Metz du 26 février au 9 juin 2014, pp. 33-41. Paris : Flammarion ; Centre Pompidou-Metz.

Coquet, J.-C. (2007). Phusis et logos. Saint-Denis : Presses universitaires de Vincennes. Eco, U. (1987). La guerre du faux. Paris : Grasset.

Fontanille, J., \& Zilberberg, C. (1998). Tension et signification. Sprimont : Mardaga.

Fresnault-Deruelle, P. (1993). L'éloquence des image : images fixes III. Paris : PUF.

Fried, M. (1990). La place du spectateur : esthétique et origines de la peinture moderne. Paris : Gallimard.

Galinon-Mélenec, B. (Dir.). (2011). L'homme trace : perspectives anthropologiques des traces contemporaines. Paris: CNRS éditions.

Goffman, E. (1973). La mise en scène de la vie quotidienne. 1. La présentation de soi. Paris : Éditions de Minuit.

Greimas, J. G., \& Fontanille, J. (1991). Sémiotique des passions : des états de choses aux états d'âme. Paris : Seuil.

Hall, E. T. (1971, éd. 2014). La dimension cachée, (trad. A. Petita), coll. Points essais. Paris : Seuil.

Heinich, N. (2012). De la visibilité : excellence et singularité en régime médiatique. Paris : Gallimard.

Heinich, N. (2014). Les paparazzis, agents du capital de visibilité. Dans Paparazzi! Photographes, stars et artistes, catalogue de l'exposition présentée au Centre Pompidou-Metz du 26 février au 9 juin 2014, pp. 98-103. Paris : Flammarion ; Centre Pompidou-Metz.

Hénault, A. (1994). Le pouvoir comme passion. Paris : PUF.

Henault, A. (2012). Les enjeux de la sémiotique. Paris : PUF.

Joly, M. (2009). Introduction à l'analyse de l'image. Paris : Armand Colin.

Landowski, E. (2005). Les interactions risquées. Nouveaux actes sémiotiques, 101-103.

Le Breton, D. (1990). Anthropologie du corps et modernité. Paris : PUF.

Paris, J. (1969). L'espace et le regard. Paris : Seuil.

Ricoeur, P. (1996). Soi-même comme un autre, coll. Point essais. Paris : Seuil.

Roche, D. (1982). La disparition des lucioles : réflexions sur l'acte photographique. Paris : Cahiers du cinéma.

Sartre, J.-P. (1938, éd. 1995). Esquisse d'une théorie des émotions. Paris : Le Livre de Poche.

Schaeffer, J.-M. (1987). L'image précaire : Du dispositif photographique. Paris : Seuil.

Schapiro, M. (2000). Face et profil comme formes symboliques. Les mots et les images (1996), (trad.). Paris : Macula.

Sorlin, P. (2000). Persona: Du portrait en peinture. Saint-Denis : Presses universitaires de Vincennes.

Todorov, T. (2000). Eloge de l'individu : Essai sur la peinture flamande de la Renaissance. Paris : A. Biro. 
Wölfflin, H. (1992). Principes fondamentaux de l'histoire de l'art, Le problème de l'évolution du style dans l'Art Moderne, (trad. par C. \& M. Raymond, éd. 1929). Paris : Gérard Monfort.

Wrona, A. (2012). Face au portrait : de Sainte-Beuve à Facebook, coll. Cultures numériques. Paris : Hermann.

Zilberberg, C. (2006). Eléments de grammaire tensive. Limoges : PULIM. 